\title{
La construcción de edificios residenciales en España en el período 2007-2010 y su impacto según el indicador Huella Ecológica
}

\author{
The construction of residential buildings in Spain in the period 2007-201O \\ and its impact according to the Ecological Footprint indicator \\ $\underline{\text { P. González-Vallejo }}^{(*)}$, J.Solís-Guzmán ${ }^{(*)}$, R. Llácer ${ }^{(*)}$, M. Marrero ${ }^{(*)}$
}

RESUMEN

Se identifican en informes estadísticos oficiales, las características principales de las viviendas construidas en España durante el período 2007 a 2010, mostrando las 10 tipologías de edificios residenciales y soluciones constructivas representativas. Se evalúa el impacto generado por dichas tipologías empleando el indicador huella ecológica (HE), obteniendo, entre otros resultados, que las viviendas unifamiliares tienen un $45 \%$ de huella superior a las plurifamiliares y que la huella que genera la mano de obra es un 35 \% de la total. También se realiza el análisis de huella por fases de obra y sistemas constructivos, que determina que los elementos de mayor impacto son, estructura, albañilería, revestimientos y cimentaciones, por ese orden. Se finaliza con el cálculo total de HE durante el período objetivo y por persona de las viviendas evaluadas.

Palabras clave: Huella ecológica; edificio residencial; metodología; recursos; energía.

\section{ABSTRACT}

This work identifies, in statistical reports, the main features of the homes built in Spain during 2007-2010; and the reports identify 10 representative types of residential buildings and construction solutions. The impact generated by these typologies is then assessed using the Ecological Footprint (EF) indicator, obtaining, among other results, that singlefamily dwellings have a $45 \%$ higher footprint than the multifamily buildings and that the manpower represents $30 \%$ of the total EF. Also, the footprint is evaluated per construction phase and construction system, which determines that the elements with higher impact are the structure, masonry, finishes and foundation, in that order. Finally, the total EF of dwelling construction during the period and per dwelling occupancy is assessed.

Keywords: Ecological footprint; residential building; methodology; resources; energy.

(*) Universidad de Sevilla (España).

Persona de contacto/Corresponding author: pgonzalez1@us.es (P. González-Vallejo)

Cómo citar este artículo/Citation: González-Vallejo, P., Solís-Guzmán, J., Llácer, R., Marrero, M. (2015). La construcción de edificios residenciales en España en el período 2007-2010 y su impacto según el indicador Huella Ecológica. Informes de la Construcción, 67(539): e111, doi: http://dx.doi.org/10.3989/ic.14.017.

Licencia / License: Salvo indicación contraria, todos los contenidos de la edición electrónica de Informes de la Construcción se distribuyen bajo una licencia de uso y distribución Creative Commons Reconocimiento no Comercial 3.o. España (cc-by-nc). 


\section{INTRODUCCIÓN}

Dentro de las actividades industriales, la actividad constructora, incluida su industria asociada, es la mayor consumidora de recursos naturales tales como madera, minerales, agua y energía. A nivel de la Unión Europea, se le considera responsable del $40 \%$ del consumo final de la energía (1). La construcción y el uso de los edificios en España suponen más del $30 \%$ del consumo de energía, principalmente no renovable, $30 \%$ de la generación de emisiones de $\mathrm{CO}_{2}$, más del $20 \%$ de las extracciones de materiales de la corteza terrestre y entre el 30 y el $40 \%$ de los residuos sólidos generados (2). A estas cifras habría que añadir las emisiones debidas a los procesos de fabricación, transporte y generación eléctrica asociados a la edificación, incluidos actualmente en otros apartados.

España superó en 2011 la cifra de 25 millones de viviendas (3), sumando viviendas principales y secundarias, cifra que a pesar de la escasa actividad de la construcción en la actualidad, no dejó de aumentar en la última década. Así, mientras que el incremento de viviendas en el período 2002 a 2008 era siempre superior al $2 \%$ anual (3), en 2010, en plena crisis del sector, fue del o,8 \% anual (3). Ese crecimiento desmesurado provocó un impacto ambiental mucho mayor que el que debería haber originado una actividad constructora más racional, y que hasta ahora no ha sido cuantificado.

Es necesario evaluar este aspecto a través de indicadores, de forma que se pueda calificar y cuantificar el peso de los impactos durante su ciclo de vida, desde la extracción de las materias primas hasta su demolición. Las herramientas disponibles generalmente siguen la metodología de Análisis de Ciclo de Vida (ACV) (4). Aparte, están surgiendo otras, como el análisis de emergía (5) o el análisis de flujo de materiales (6). Sin embargo, no existen aproximaciones estandarizadas desde el punto de vista de otras metodologías, como la huella ecológica.

El indicador huella ecológica (HE) fue introducido por Mathis Wackernagel (7), que midió la HE de la humanidad y la comparó con la capacidad de carga del planeta. De acuerdo con su definición, la HE es la extensión de tierra que sería necesaria para suministrar los recursos (cereales, pienso, leña, pescado y terreno urbano) y absorber las emisiones $\left(\mathrm{CO}_{2}\right)$ de la sociedad mundial (8). Actualmente es considerado uno de los indicadores más relevantes para evaluar los impactos generados en el medio ambiente, y además puede ser usado conjuntamente con otros indicadores, como la huella de carbono (9) o la huella hídrica (10). El indicador se ha usado desde sus inicios para determinar impactos a muy diversa escala: para predecir los impactos que generará la humanidad sobre el Planeta Tierra, para realizar el cálculo periódico de la huella que la humanidad marca, o también para calcular periódicamente las huellas de los distintos países, ciudades, barrios, sectores productivos o industrias (11).

El objetivo principal del trabajo es la evaluación de los edificios residenciales construidos entre 2007 y 2010 mediante el indicador HE, empleando la metodología de cálculo desarrollada previamente por los autores (11) (12) en 2011 y mejorada en 2015 (13) con aportaciones como, la creación de un banco de cuantificación de recursos y la evaluación del consumo de agua mediante el estudio de 90 proyectos de construcción. Avanzando en la estandarización y aplicabilidad del anterior modelo, partiendo del presupuesto, la medición y datos gene- rales del proyecto y apoyado en la Base de Costes de Construcción de Andalucía (BCCA) (14) (15), en el presente trabajo, por primera vez, se desagrega la información en materiales, mano de obra y maquinaria, y en las fases del proyecto. Así, se evalúa la HE por capítulos del proyecto y se identifican los elementos que controlan la huella en cada etapa constructiva, obteniéndose además las soluciones constructivas con mayor impacto. Finalmente, y partiendo de los resultados previos, se obtiene el cálculo total de HE en España y por persona de las viviendas evaluadas.

Para aplicar el modelo HE a la construcción en España en el período 2007 a 2010, se parte de estudios estadísticos (16) que definen las tipologías de viviendas construidas en dicho período.

\section{El procedimiento de trabajo seguido es:}

- Identificación de las características de los edificios residenciales construidos en España en el período 2007-2010 (16).

- Selección de las 10 tipologías edificatorias más representativas de ese período y búsqueda de proyectos reales.

- Creación de un banco de cuantificación de recursos (BCRR), para las 10 tipologías.

- Aplicación del indicador HE.

- Análisis de los resultados.

\section{METODOLOGÍA}

\subsection{Análisis tipológico de la edificación residencial en España (2007-2010)}

Se ha realizado estudio estadístico para identificar: tipologías representativas, características constructivas, patrones de uso, etc. Las diferentes climatologías y topografías hacen que no resulte fácil unificar las tipologías constructivas. Por tanto, seleccionar materiales y sistemas constructivos representativos en España es una tarea que exige un análisis de los datos estadísticos publicados. Para ello se analizaron distintas fuentes documentales, siendo el informe La construcción de edificios en España (2006-2010) del Ministerio de Fomento (16) la más completa. Los datos de esta fuente proceden de las licencias municipales de obra mayor concedidas por los ayuntamientos, y las hojas de estadísticas de las que se obtienen los datos constan de dos apartados fundamentales para el presente estudio:

- Datos generales de la obra y del promotor, cómo el número de edificios construidos según uso, tipologías y presupuesto. - Edificación de nueva planta: características generales, tipología constructiva, instalaciones, energía utilizada y características de las viviendas.

Presentaremos la información tabulada de los siguientes apartados:

- Tipo de obra, según uso y tipología.

- Características generales de los edificios.

- Tipología constructiva, instalaciones y características de las viviendas.

Según el tipo de obra la clasificación es la siguiente: rehabilitación, demolición o nueva planta. En este caso se estudiaron los edificios de uso residencial, que se clasifican en: destinados a vivienda familiar, bien unifamiliar (aislados, adosados 
o pareados) o plurifamiliar (con dos o más viviendas) o a residencia colectiva (permanente o eventual). Según el análisis estadístico (16), los edificios a rehabilitar y demoler entre 2006 a 2010 suman una superficie construida total $\left(50.670 \mathrm{~m}^{2}\right)$ muy inferior a la de los de nueva planta $\left(402.379 \mathrm{~m}^{2}\right)$. De estos últimos, los residenciales $\left(312.212 \mathrm{~m}^{2}\right)$ representan, según la superficie construida total, el $78 \%$, lo que justifica que se haya centrado el estudio en este tipo de edificios de nueva planta.

De las características recogidas en los estudios estadísticos (16) la más importante para el presente análisis es el número de plantas sobre y bajo rasante, ya que dicha característica define en gran medida las soluciones constructivas, siendo un factor determinante para la HE de cada edificio. Se identifica (Tabla 1) un mayor porcentaje de edificios entre 4 y 5 plantas sobre rasante y los menos representativos son los de o o 1 planta.

Se recogen los porcentajes de los edificios de nueva planta de uso residencial según tipología constructiva, instalaciones y acabado interior, referentes a cada año desde 2007 a 2010, siendo todos los años los datos muy similares (16), por lo que se han representado los valores medios en la Tabla 2.

\subsection{Selección de tipologías representativas y proyectos reales}

A partir de los datos analizados en la Tabla 2, y de acuerdo a los porcentajes que representa cada elemento sobre el total de cada parte en que se divide el edificio o vivienda, se obtiene la propuesta de las 10 viviendas tipo (Tablas 3 y 4).

Tabla 1. Número de viviendas construidas en España según el número de plantas sobre rasante del edificio (2007-2010).

\begin{tabular}{|c|c|c|c|c|c|c|c|c|c|}
\hline \multirow{3}{*}{\multicolumn{2}{|c|}{$\begin{array}{c}\text { Número } \\
\text { de plantas }\end{array}$}} & \multicolumn{8}{|c|}{ Año } \\
\hline & & \multicolumn{2}{|c|}{2007} & \multicolumn{2}{|c|}{2008} & \multicolumn{2}{|c|}{2009} & \multicolumn{2}{|c|}{2010} \\
\hline & & Total & $\%$ & Total & $\%$ & Total & $\%$ & Total & $\%$ \\
\hline \multirow{6}{*}{$\begin{array}{l}\text { Plantas } \\
\text { sobre } \\
\text { rasante }\end{array}$} & o y 1 & 23.572 & 4 & 14.042 & 5 & 7.759 & 6 & 7.479 & 8 \\
\hline & 2 & 151.902 & 24 & 63.913 & 24 & 27.668 & 21 & 24.101 & 26 \\
\hline & 3 & 144.723 & 23 & 52.694 & 20 & 25.014 & 19 & 13.631 & 15 \\
\hline & 4 y 5 & 210.065 & 33 & 86.744 & 32 & 38.515 & 30 & 25.661 & 28 \\
\hline & 6 o más & 103.836 & 16 & 51.042 & 19 & 31.590 & 24 & 20.773 & 23 \\
\hline & TOTAL & 634098 & 100 & 268435 & 100 & 130546 & 100 & 91645 & 100 \\
\hline \multirow{4}{*}{$\begin{array}{l}\text { Plantas bajo } \\
\text { rasante }\end{array}$} & $\mathbf{0}$ & 141.777 & 22 & 66.840 & 22 & 30.507 & 23 & 25.216 & 28 \\
\hline & $\mathbf{1}$ & 328.920 & 52 & 121.018 & 52 & 59.321 & 45 & 37.288 & 41 \\
\hline & 20 más & 163.401 & 26 & 80.577 & 26 & 29.141 & 31 & 29.141 & 32 \\
\hline & TOTAL & 634098 & 100 & 268435 & 100 & 118969 & 100 & 91645 & 100 \\
\hline
\end{tabular}

Tabla 2. Porcentajes de edificios de nueva planta de uso residencial según características constructivas.

\begin{tabular}{|c|c|c|c|c|c|c|c|}
\hline \multicolumn{8}{|c|}{ Características constructivas de edificios residenciales (\%) } \\
\hline \multicolumn{4}{|c|}{ Estructura vertical } & \multicolumn{2}{|c|}{ Estructura horizontal } & \multicolumn{2}{|c|}{ Cubierta } \\
\hline $\begin{array}{l}\text { Hormigón } \\
\text { armado }\end{array}$ & Metálica & $\begin{array}{l}\text { Muros } \\
\text { de carga }\end{array}$ & Mixta y otros & Unidirec. & Losa & Plana & Inclinada \\
\hline 73 & 6 & 15 & 6 & 84 & 16 & 33 & 67 \\
\hline \multicolumn{4}{|c|}{ Cerramiento exterior } & \multicolumn{4}{|c|}{ Carpintería exterior } \\
\hline Cerámico & Pétreos & $\begin{array}{l}\text { Revestim. } \\
\text { continuo }\end{array}$ & Otros & Madera & Alum. & Plástico & $\begin{array}{c}\text { Chapa de } \\
\text { acero y otros }\end{array}$ \\
\hline 50 & 12 & 34 & 4 & 8 & 84 & 7 & 1 \\
\hline \multicolumn{8}{|c|}{ Instalaciones } \\
\hline $\begin{array}{l}\text { Evacuac. } \\
\text { aguas } \\
\text { residuales }\end{array}$ & $\begin{array}{l}\text { Suministro } \\
\text { agua potable }\end{array}$ & $\begin{array}{l}\text { Agua } \\
\text { caliente }\end{array}$ & Calefac. & Refriger. & $\begin{array}{c}\text { Ascensores } \\
\text { y/o } \\
\text { montacargas }\end{array}$ & $\begin{array}{c}\text { Tratam. } \\
\text { aguas } \\
\text { residuales }\end{array}$ & $\begin{array}{c}\text { Tratam. } \\
\text { otros } \\
\text { residuos }\end{array}$ \\
\hline 100 & 100 & 96 & 65 & 18 & 68 & 7 & 2 \\
\hline \multicolumn{8}{|c|}{ Acabado Interior } \\
\hline \multicolumn{4}{|c|}{ Solería } & \multicolumn{2}{|c|}{ Carpintería Interior } & \multirow{2}{*}{$\begin{array}{c}\text { Con falso } \\
\text { techo }\end{array}$} & \multirow{2}{*}{$\begin{array}{c}\text { Con persia- } \\
\text { nas }\end{array}$} \\
\hline Cerámico & Pétreo & Madera & Otros & Madera & Otros & & \\
\hline 53 & 15 & 29 & 3 & 98 & 2 & 76 & 93 \\
\hline
\end{tabular}


Hay características que son comunes en todas las tipologías, como:

- La carpintería exterior es de aluminio y tienen persianas.

- La carpintería interior es de madera.

- Las instalaciones de evacuación de aguas residuales, suministro de agua potable y agua caliente sanitaria se colocan en todas las viviendas, sin embargo ninguna tiene un sistema de tratamiento de residuos.

- La energía más utilizada es la electricidad.

Las demás características varían en función de cada tipología y se definen en las Tablas 3 y 4.

Algunos de los datos porcentuales para definir las 10 tipologías (16) son: las plurifamiliares (2 o más viviendas) representan el $75 \%$ y las unifamiliares el $25 \%$ (10 \% aisladas, $13 \%$ adosadas y $2 \%$ pareadas). Según el número de plantas sobre rasante, el $6 \%$ son de una planta, $24 \%$ son de dos, repartido entre unifamiliares y plurifamiliares. De tres o más plantas son todas plurifamiliares: $18 \%$ de tres, $31 \%$ de cuatro y cinco y $21 \%$ de 6 o más.

Según el número de plantas bajo rasante y plurifamiliares tienen una $45 \%$, y dos $30 \%$. Las unifamiliares, que son el restante $25 \%$, no disponen de sótano.

Se realiza una búsqueda de 10 proyectos reales que se correspondan con las tipologías de viviendas más construidas en España en los años 2007 a 2010, mostradas en las Tablas 3 y 4 .

\subsection{Metodología para el cálculo de HE}

Para el cálculo de la HE los datos del proyecto se clasifican en:

- Datos procedentes de las mediciones y presupuesto: Las cantidades (Qi) de cada proyecto están estructuradas siguiendo la clasificación sistemática del BCCA (15), y se expresan en unidades de medición por unidad de superficie construida $\left(\mathrm{u} / \mathrm{m}^{2}\right)$. En la Figura 1 se muestra, a modo de ejemplo, el apartado 05F Forjados que pertenece al capítulo 05 Estructuras. Para el resto de apartados y capítulos del presupuesto se sigue el mismo criterio, para lo cual se crea el Banco de Cuantificación de Recursos (BCRR), del que se obtiene la cantidad de recursos utilizados en la obra, descompuestos en mano de obra, materiales y maquinaria, tomando valores medios $\left(\mathrm{Q}_{\mathrm{m}}\right)$ a partir de soluciones constructivas representativas (en dicha figura se representan las soluciones de las que se obtienen los valores medios). Se muestra también, para el caso del forjado de bovedillas, los valores medios de materiales, mano de obra y maquinaria. Partiendo del valor de $\mathrm{Q}_{\mathrm{i}}$, que es único para cada apartado de cada capítulo del proyecto, y del $\mathrm{Q}_{\mathrm{m}}$ de cada elemento, la cantidad total $\left(Q_{t}\right)$ de cada recurso empleado se obtiene de la siguiente forma:

$$
\mathrm{Q}_{\mathrm{t}}=\mathrm{Q}_{\mathrm{i}} \times \mathrm{Q}_{\mathrm{m}} \times \mathrm{S}
$$

donde:

S es la superficie construida total por vivienda.

Tabla 3. Propuesta de tipología de viviendas (1 a 5).

\begin{tabular}{|c|c|c|c|c|c|c|}
\hline \multicolumn{7}{|c|}{ Propuesta de viviendas 1-5 } \\
\hline \multirow{2}{*}{\multicolumn{2}{|c|}{ CARACTERÍSTICAS }} & \multicolumn{5}{|c|}{ CODIFICACIÓN DE TIPOLOGÍA DE VIVIENDAS } \\
\hline & & $\mathbf{1}$ & 2 & 3 & 4 & 5 \\
\hline \multicolumn{2}{|c|}{ Tipo de residencia } & \multicolumn{2}{|c|}{ UNIFAMILIAR } & \multicolumn{3}{|c|}{ PLURIFAMILIAR } \\
\hline \multicolumn{2}{|c|}{ Tipología constructiva } & Aislada & Adosada & \multicolumn{3}{|c|}{ Dos o más viviendas } \\
\hline \multicolumn{2}{|l|}{ Superficie útil } & 181 & 143 & \multicolumn{3}{|c|}{72} \\
\hline \multicolumn{2}{|c|}{ No de plantas sobre rasante } & 1 & 2 & 2 & 3 & 3 \\
\hline \multicolumn{2}{|c|}{ No de plantas bajo rasante } & o & o & 1 & 1 & 1 \\
\hline \multirow{4}{*}{$\begin{array}{l}\text { Características } \\
\text { constructivas }\end{array}$} & Estructura vertical & Muros de carga & \multicolumn{4}{|c|}{ Hormigón } \\
\hline & Estructura horizontal & Losa & Losa & \multicolumn{3}{|c|}{ Unidireccional } \\
\hline & Cubierta & Inclinada & Plana & Plana & Inclinada & Plana \\
\hline & Cerramiento exterior & Revestimiento & Pétreo & Otros & Cerámico & Cerámico \\
\hline \multirow{2}{*}{$\begin{array}{l}\text { Acabado } \\
\text { interior }\end{array}$} & Solería & \multicolumn{2}{|c|}{ Cerámica } & Pétrea & Madera & Cerámica \\
\hline & Falso techo & \multicolumn{2}{|c|}{ No } & \multicolumn{3}{|c|}{ Sí } \\
\hline \multicolumn{2}{|c|}{ Número de habitaciones } & \multicolumn{2}{|c|}{6} & \multicolumn{3}{|c|}{4} \\
\hline \multicolumn{2}{|l|}{ Número de baños } & 3 & \multicolumn{4}{|c|}{2} \\
\hline \multirow{4}{*}{$\begin{array}{l}\text { Instalaciones } \\
\text { existentes }\end{array}$} & Calefacción & & Sí & & No & Sí \\
\hline & Refrigeración & Sí & \multicolumn{4}{|c|}{ No } \\
\hline & Ascensor o montacargas & \multicolumn{3}{|c|}{ No } & \multicolumn{2}{|c|}{ Sí } \\
\hline & Tratamiento de aguas residuales & \multicolumn{5}{|c|}{ No } \\
\hline \multirow{2}{*}{$\begin{array}{l}\text { Energía } \\
\text { instalada }\end{array}$} & Gas ciudad o natural & No & Sí & No & Sí & No \\
\hline & Energía solar & Sí & No & Sí & No & Sí \\
\hline \multicolumn{2}{|l|}{ Plazas de garaje } & \multicolumn{2}{|c|}{ No } & \multicolumn{3}{|c|}{ Sí } \\
\hline \multicolumn{2}{|l|}{ Promoción } & Uso propio & $\begin{array}{l}\text { Sociedad } \\
\text { mercantil }\end{array}$ & Privado & $\begin{array}{c}\text { Cooperativa y } \\
\text { otros }\end{array}$ & $\begin{array}{l}\text { Sociedad } \\
\text { mercantil }\end{array}$ \\
\hline
\end{tabular}


En la Figura 1, $\mathrm{Q}_{\mathrm{i}}$ se mide en $\mathrm{m}^{2}$ de forjado $/ \mathrm{m}^{2}$ de superficie construida, los Qm se miden en unidades de medición del recurso $/ \mathrm{m}^{2}$ de forjado, por lo que los $\mathrm{Q}_{\mathrm{t}}$ quedan en la unidad del recurso considerado $\left(\mathrm{kg}, \mathrm{m}^{2}, \mathrm{~m}^{3}\right.$, etc.). Los $\mathrm{Q}_{\mathrm{t}}$ obtenidos generan las distintas huellas que conforman la HE (Figuras 1 y 2).

- Datos generales del proyecto: presupuesto total de obra (€), año de construcción, superficie construida $\left(\mathrm{m}^{2}\right)$, energía consumida en obra $(€)$ y número de plantas sobre rasante.

A continuación se describe resumidamente cada de las huellas parciales que se obtienen según la metodología expuesta en la Figura 2, bien de las mediciones y presupuesto de proyecto (alimentos, residuos sólidos urbanos (RSU) y movilidad de la mano de obra, materiales y maquinaria) o de los datos generales (residuos de construcción y demolición (RCD), electricidad, agua y superficie consumida).

2.3.1. Huella procedente de los datos del presupuesto

\subsubsection{Huella derivada de la mano de obra}

Según la Figura 2, la huella de los trabajadores procede de la alimentación, RSU y movilidad.

Se imputa la manutención de los trabajadores a la huella de la edificación ya que dicha actividad se desarrolla en el interior de la obra, de forma similar a la metodología desarrollada por Domenech Quesada (17). Para ello, en primer lugar es necesario conocer el número total de horas trabajadas a través del BCRR.
Para el cálculo de la huella empleamos la expresión,

$$
\mathrm{HE}_{\mathrm{pal}}=\frac{\mathrm{HE}_{\mathrm{c}}}{\mathrm{h}_{\mathrm{c}}} \mathrm{N}_{\mathrm{h}}
$$

donde:

$\mathrm{HE}_{\mathrm{pal}}$ : HE ponderada de los alimentos (hag) (cuatro tipos: energía, pastos, mar y cultivos)

HE : HE expresada como hag/comida, definida en la metodología (10) (12)

$\mathrm{h}_{\mathrm{c}}: 8$ horas/comida. Se considera 1 única comida por cada jornada de trabajo

$\mathrm{N}_{\mathrm{h}}$ : número total de horas trabajadas

Los valores de $\mathrm{HE}_{\mathrm{c}}$ (hag/comida) para un menú tipo de trabajador son calculados de acuerdo a Solís-Guzmán et al. (11): energía 0,0072, pastos 0,0234, mar 0,0159 y cultivo 0,0085.

Para determinar la HE relacionada con la movilidad de los trabajadores se seguirán las hipótesis establecidas en el método (11) (12): transporte del operario desde casa hasta la obra, ocupación media de vehículo 4 personas y distancia media recorrida por día $30 \mathrm{~km}$. (ida y vuelta). Habrá que conocer el número de trabajadores, el número total de horas trabajadas y la duración efectiva de la obra en horas. Estos datos se obtendrán de la medición y de los rendimientos totales obtenidos del BCRR.

Para el cálculo del combustible emplearemos coeficientes de consumo de coches en España, que en el caso del gasoil es $6,8 \mathrm{l} / 100 \mathrm{~km} \mathrm{(18).}$

Tabla 4. Propuesta de tipología de viviendas (6 a 10).

\begin{tabular}{|c|c|c|c|c|c|c|}
\hline \multicolumn{7}{|c|}{ Propuesta de viviendas 6-10 } \\
\hline \multirow{2}{*}{\multicolumn{2}{|c|}{ CARACTERÍSTICAS }} & \multicolumn{5}{|c|}{ CODIFICACIÓN DE TIPOLOGÍA DE VIVIENDAS } \\
\hline & & 6 & 7 & 8 & 9 & 10 \\
\hline \multicolumn{2}{|c|}{ Tipo de residencia } & \multicolumn{5}{|c|}{ PLURIFAMILIAR } \\
\hline \multicolumn{2}{|c|}{ Tipología constructiva } & \multicolumn{5}{|c|}{ Dos o más viviendas } \\
\hline \multicolumn{2}{|c|}{ Superficie útil } & 70 & \multicolumn{4}{|c|}{72} \\
\hline \multicolumn{2}{|c|}{$N^{o}$ de plantas sobre rasante } & 4 & 5 & 5 & 6 o más & 6 o más \\
\hline \multicolumn{2}{|c|}{$N^{\circ}$ de plantas bajo rasante } & 2 & 1 & 2 & 1 & 2 \\
\hline \multirow{4}{*}{$\begin{array}{l}\text { Características } \\
\text { constructivas }\end{array}$} & Estructura vertical & \multicolumn{5}{|c|}{ Hormigón } \\
\hline & Estructura horizontal & Losa & \multicolumn{4}{|c|}{ Unidireccional } \\
\hline & Cubierta & \multicolumn{2}{|c|}{ Plana } & Inclinada & Plana & Inclinada \\
\hline & Cerramiento exterior & Revestimiento & Pétreo & Otros & \multicolumn{2}{|c|}{ Cerámico } \\
\hline \multirow{2}{*}{$\begin{array}{l}\text { Acabado } \\
\text { interior }\end{array}$} & Solería & Pétrea & Cerámica & Madera & Cerámica & Madera \\
\hline & Falso techo & \multicolumn{5}{|c|}{ Sí } \\
\hline \multicolumn{2}{|c|}{ Número de habitaciones } & 6 & \multicolumn{4}{|c|}{4} \\
\hline \multicolumn{2}{|c|}{ Número de baños } & \multicolumn{5}{|c|}{2} \\
\hline \multirow{4}{*}{$\begin{array}{l}\text { Instalaciones } \\
\text { existentes }\end{array}$} & Calefacción & $\mathrm{S}$ & & No & Sí & No \\
\hline & Refrigeración & \multicolumn{3}{|c|}{ No } & Sí & No \\
\hline & Ascensor o montacargas & \multicolumn{5}{|c|}{ Sí } \\
\hline & Tratamiento de aguas residuales & \multicolumn{4}{|c|}{ No } & Sí \\
\hline \multirow{2}{*}{$\begin{array}{l}\text { Energía } \\
\text { instalada }\end{array}$} & Gas ciudad o natural & \multicolumn{2}{|c|}{ No } & Sí & No & Sí \\
\hline & Energía solar & \multicolumn{2}{|c|}{ Sí } & No & Sí & No \\
\hline \multicolumn{2}{|l|}{ Plazas de garaje } & \multicolumn{5}{|c|}{ Sí } \\
\hline \multicolumn{2}{|l|}{ Promoción } & \multicolumn{5}{|c|}{ Sociedad mercantil } \\
\hline
\end{tabular}


La HE del consumo de combustible es,

$$
\mathrm{HE}_{\mathrm{pc}}=\frac{\mathrm{C}}{\mathrm{PE}} \mathrm{FE}_{\mathrm{B}}
$$

donde:

$\mathrm{HE}_{\mathrm{pc}}$ :HE ponderada del consumo de combustible (hag)

C: consumo de combustible (GJ). Intensidad energética del petróleo: $35 \mathrm{MJ} / \mathrm{l}$ (19)

$\mathrm{FE}_{\mathrm{B}}$ : factor de equivalencia de los bosques (1,26 hag/ha) (20)

PE: productividad energética del combustible (GJ/ha), que se considera la del petróleo (Tabla 5). La productividad energética de un combustible se expresa como,

$$
\mathrm{PE}=\frac{\mathrm{F}_{\mathrm{a}}}{\mathrm{F}_{\mathrm{e}}}
$$

donde:

$\mathrm{F}_{\mathrm{a}}$ es el factor de absorción de bosques de 5,21 $\mathrm{t} \mathrm{CO}_{2} /$ ha y año (17)

$\mathrm{F}_{\mathrm{e}}$ es el factor de emisión del petróleo (Tabla 5)
Los RSU provienen de la actividad de la mano de obra. A partir del número de trabajadores de cada proyecto, datos estadísti$\cos$ (21) (22) y la intensidad energética de los materiales (12), se generan coeficientes intermedios mostrados en la Tabla 6.

Tabla 5. Factores de emisión, $F_{e}$, (19) y productividades energéticas, $\mathrm{PE}$, de distintas fuentes de energía.

\begin{tabular}{|l|c|c|}
\hline \multicolumn{1}{|c|}{ Fuentes de energía } & $\mathbf{F}_{\mathbf{e}}$ (kg C/GJ) & PE (GJ/ha) \\
\hline Carbón & $\mathbf{2 6}$ & 55 \\
\hline Petróleo & $\mathbf{2 0}$ & 71 \\
\hline Gas natural & 15,3 & 93 \\
\hline Nuclear & 20 & 71 \\
\hline
\end{tabular}

Tabla 6. Índices de conversión ponderados para RSU.

\begin{tabular}{|l|c|c|c|c|}
\hline \multicolumn{1}{|c|}{ Índices } & Orgánico & Papel & Plástico & Vidrio \\
\hline $\mathrm{IC}_{\mathrm{i}}$ (hag/año/t) & 0,31 & 1,15 & 0,56 & 0,30 \\
\hline $\mathrm{G}_{\mathrm{i}}(\mathrm{t} /$ trabajador/año $)$ & 0,25 & 0,12 & 0,06 & 0,04 \\
\hline
\end{tabular}

\begin{tabular}{|c|c|c|c|c|c|c|c|c|}
\hline \multirow[b]{2}{*}{$\begin{array}{l}\text { Recursos } \\
\text { empleados }\end{array}$} & \multicolumn{6}{|c|}{ Precios procedentes de distintas soluciones constructivas } & \multirow[b]{2}{*}{$\begin{array}{l}\text { Cantidades } \\
\text { medias } \\
(Q m)\end{array}$} & \multirow[b]{2}{*}{$\begin{array}{c}\text { Cantidades } \\
\text { totales } \\
\left(Q t=Q^{*} Q^{*} \text { Sup }\right)\end{array}$} \\
\hline & Unidades & $\begin{array}{c}\text { 05FUA } \\
00018\end{array}$ & $\begin{array}{c}\text { 05FUA } \\
00118\end{array}$ & $\begin{array}{l}\text { 05FUS } \\
00018\end{array}$ & $\begin{array}{c}\text { 05FUS } \\
00108\end{array}$ & $\begin{array}{c}\text { 05FUS } \\
00118\end{array}$ & & \\
\hline
\end{tabular}

05F.02. m2. Forjado de bovedillas de mortero de cemento

\begin{tabular}{|c|c|c|c|c|c|c|c|c|}
\hline Acero B500S & $\mathrm{kg}$ & 1,99 & 2,99 & 2,19 & 3,49 & 3,49 & 2,83 & $18661,00 \mathrm{~kg}$ \\
\hline $\begin{array}{l}\text { Bovedilla de } \\
\text { mortero cemento }\end{array}$ & $\mathrm{u}$ & 5,40 & 4,86 & 5,40 & 4,86 & 4,64 & 5,03 & $33174,38 u$ \\
\hline $\begin{array}{l}\text { Hormigón sum. } \\
\text { HA25/P/20/lla }\end{array}$ & $\mathrm{m}^{3}$ & 0,11 & 0,12 & 0,11 & 0,12 & 0,12 & 0,11 & $751,72 \mathrm{~m}^{3}$ \\
\hline $\begin{array}{l}\text { Madera de pino } \\
\text { en tablón }\end{array}$ & $\mathrm{m}^{3}$ & 0,001 & 0,001 & 0,001 & 0,001 & 0,001 & 0,001 & $6,59 \mathrm{~m}^{3}$ \\
\hline $\begin{array}{l}\text { Vigueta autorr. } \\
\text { pretensada }\end{array}$ & $\mathrm{m}$ & 1,40 & 2,34 & 1,40 & 2,34 & 2,34 & 1,96 & $12934,78 \mathrm{~m}$ \\
\hline Pequeño material & u & 1,00 & 1,00 & 1,00 & 1,00 & 1,00 & 1,00 & $6593,99 \mathrm{u}$ \\
\hline
\end{tabular}

MATERIALES

MANO DE OBRA

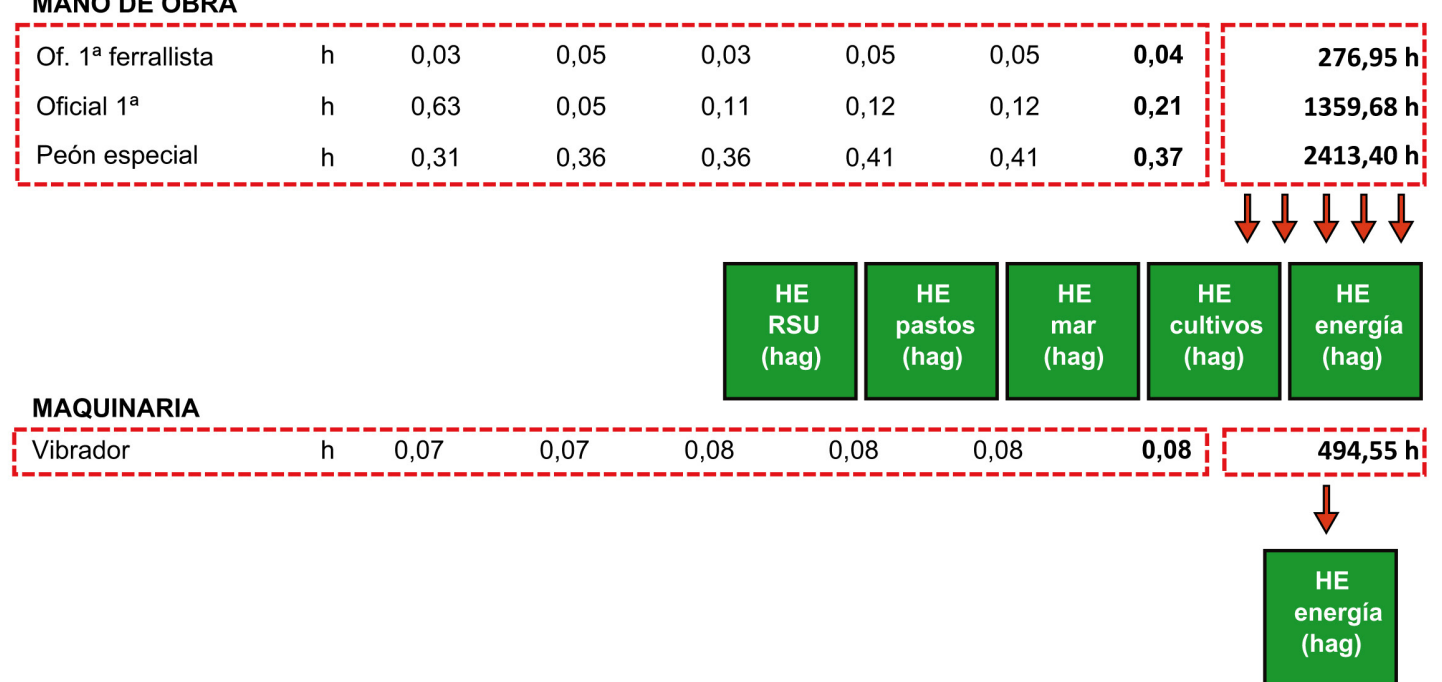

Figura 1. Esquema de cálculo del BCRR. 
Finalmente, la siguiente expresión es empleada para la determinación de la HE de los RSU,

$$
\mathrm{HE}_{\mathrm{pr}}=\sum_{\mathrm{i}} \mathrm{IC}_{\mathrm{i}} \mathrm{G}_{\mathrm{i}}
$$

donde:

$\mathrm{HE}_{\mathrm{pr}}$ : HE ponderada de los residuos (hag/año)

$\mathrm{IC}_{\mathrm{i}}:$ índice de conversión ponderado (hag/t)

$\mathrm{G}_{\mathrm{i}}$ : generación de RSU ( $\mathrm{t}$ )

\subsubsection{Huella de los materiales de construcción}

Para determinar la huella de los materiales de construcción, que se imputa a energía (Figura 2), se emplea la siguiente expresión,

$$
\mathrm{HE}_{\mathrm{pm}}=\frac{\sum_{\mathrm{i}} \mathrm{Cm}_{\mathrm{i}} \text { Eiem }_{\mathrm{i}}}{\mathrm{PE}} \mathrm{FE}_{\mathrm{B}}
$$

donde:

$\mathrm{HE}_{\mathrm{pm}}$ : HE ponderada de materiales de construcción (hag)

$\mathrm{Cm}_{\mathrm{i}}$ : consumo del material $(\mathrm{kg})$

Eiem $_{\mathrm{i}}$ : energía incorporada específica del material i $(\mathrm{MJ} / \mathrm{kg})$

PE: productividad energética del petróleo $(\mathrm{MJ} / \mathrm{ha})$

Realizando el análisis con todos los materiales de obra del BCRR y su energía incorporada (Eiem) (23), se determina la energía total y su respectiva huella que se transforma a hag de absorción de bosques (1,26 hag/ha) (20).

\subsubsection{Huella de la maquinaria}

Para determinar el consumo de combustible de la maquinaria en la obra (en volumen) se obtiene partiendo del BCRR, el coste total que corresponde al sumatorio de las horas de cada máquina empleada por su correspondiente coste.

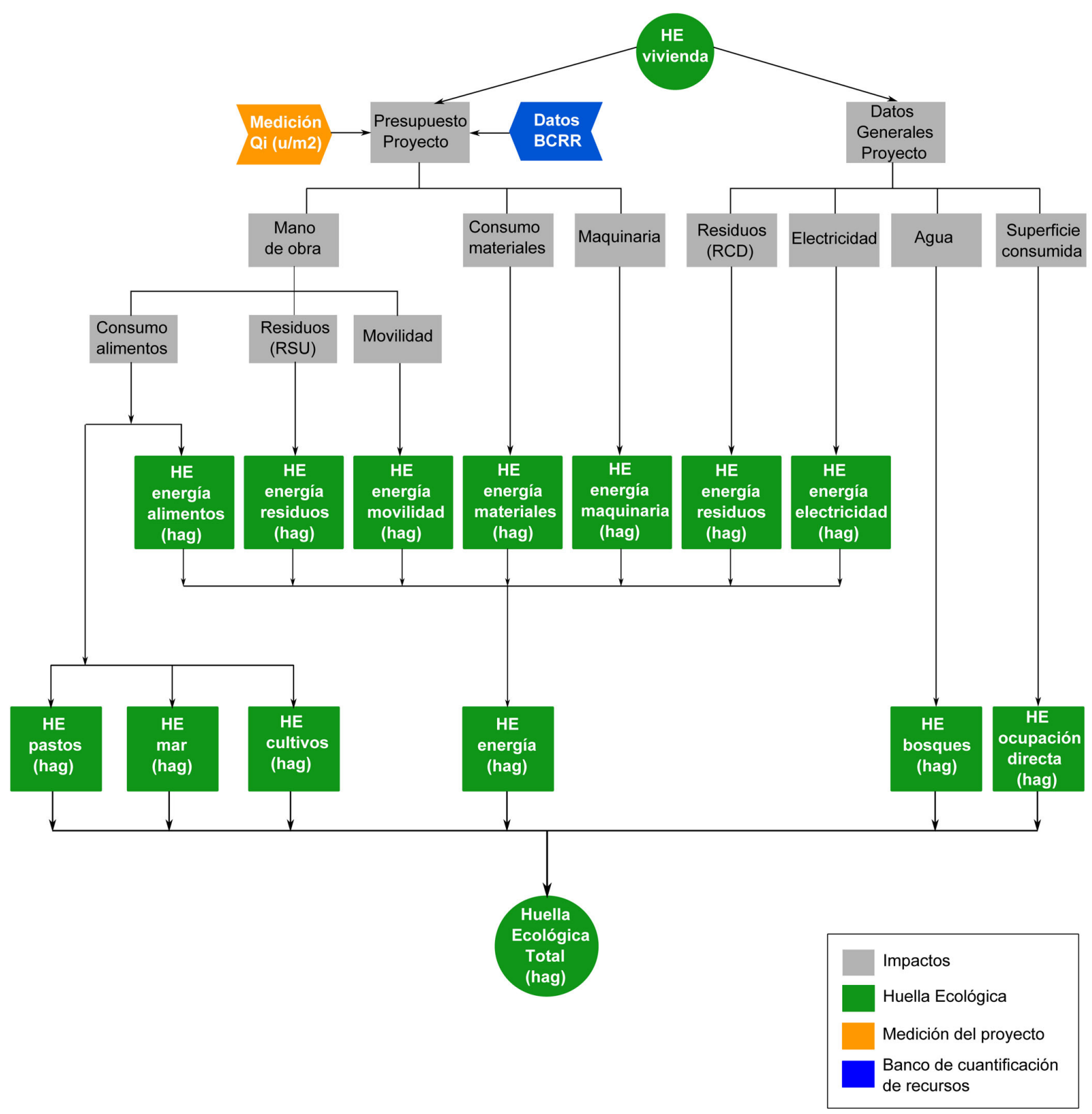

Figura 2. Árbol metodológico de la investigación (13). 
Como hipótesis, el $20 \%$ de dicho coste es considerado de combustible, que incluye el mantenimiento pero no su depreciación. El precio actual en España es 1,365 €/1 (24). La huella se determina usando el procedimiento explicado en la sección de movilidad.

\subsubsection{Huella procedente de los datos generales}

La huella generada por los datos generales del proyecto (Figura 2) procede del consumo de electricidad, generación de $\mathrm{RCD}$, consumo de agua y superficie construida.

\subsubsection{Huella de electricidad}

Para el cálculo de la electricidad, se emplea la metodología previa (11) (12), que considera que la energía de la obra se divide en combustible para maquinaria y electricidad de obra. Para predecir el consumo de energía de una obra se parte de los datos que proporcionan las fórmulas polinómicas (12). La energía eléctrica del mix eléctrico español está formada por: 23,9 \% carbón, 23,8 \% petróleo, 31,5 \% gas, 17 \% energía nuclear y el resto de fuentes renovables (25). Cada tipo de fuente tiene una productividad energética distinta (Tabla 5). El factor de eficiencia para producción de electricidad es 0,3 (18).

La huella de consumo energético es,

$$
\mathrm{HE}_{\text {pee }}=\sum_{\mathrm{i}} \frac{\mathrm{C}_{\mathrm{i}}}{\mathrm{PE}_{\mathrm{i}}} \mathrm{FE}_{\mathrm{B}}
$$

donde:

$\mathrm{HE}_{\text {pee }}$ : HE ponderada del consumo de energía eléctrica (hag) $\mathrm{C}_{\mathrm{i}}$ : Consumo de energía primaria de la fuente i (GJ) $\mathrm{PE}_{\mathrm{i}}$ : Productividad energética de la fuente i (GJ/ha)

\subsubsection{Huella de los RCD}

El cálculo de la huella de los RCD se basa en los estudios de Wackernagel (26), donde todos los consumos se imputan a la huella energética, y emplea una herramienta informática (27), que diferencia dos tipos de RCD, tierras y mixtos, (Tabla 7). El procedimiento determina índices de conversión (28) ya incorporados en las investigaciones de Domenech (17):

$$
\mathrm{IC}_{\mathrm{x}}=\frac{\mathrm{IE}_{\mathrm{x}}}{\mathrm{PE}}\left(1-\frac{\% \mathrm{R}_{\mathrm{x}}}{100} \frac{\% \mathrm{SE}_{\mathrm{x}}}{100}\right) \mathrm{FE}_{\mathrm{B}}
$$

donde :

$\mathrm{IC}_{\mathrm{x}}$ : índice de conversión ponderado del residuo no peligroso (hag/t)

$\mathrm{IE}_{\mathrm{x}}$ : intensidad energética de producción del material del que está fabricado el residuo (GJ/t) (Tabla 7)

PE: productividad energética del residuo (se asume que es igual a la de los combustibles fósiles) (GJ/ha)

$\% \mathrm{R}_{\mathrm{x}}$ : porcentaje de reciclaje de $\mathrm{x}$

$\% \mathrm{SE}_{\mathrm{x}}$ : porcentaje de energía recuperada por reciclaje (Tabla 7)

Tabla 7. Parámetros para el cálculo de los índices de conversión.

\begin{tabular}{|l|c|c|}
\hline \multicolumn{1}{|c|}{ Índices } & $\begin{array}{c}\text { Tierras } \\
\text { excavadas }\end{array}$ & RCD mixtos \\
\hline IE $_{\mathbf{x}}$ (GJ/t) & 0,1 & 5 \\
\hline PE (GJ/ha) & 71 & 71 \\
\hline \% $_{\mathbf{x}}$ & 100 & 70 \\
\hline \%SE $_{\mathbf{x}}$ & 90 & 90 \\
\hline
\end{tabular}

Finalmente, para determinar la huella total de los residuos se usará la expresión,

$$
\mathrm{HE}_{\mathrm{pr}}=\sum_{\mathrm{i}} \mathrm{IC}_{\mathrm{x}_{\mathrm{i}}} \mathrm{C}_{\mathrm{i}}
$$

donde:

$\mathrm{HE}_{\mathrm{pr}}$ : HE ponderada de los residuos (hag)

$\mathrm{IC}_{\mathrm{xi}}{ }_{\mathrm{i}}$ índice de conversión ponderado (hag/t)

$\mathrm{C}_{\mathrm{i}}$ : consumo $(\mathrm{t})$

\subsubsection{Huella derivada del consumo de agua}

Para la huella derivada del consumo de agua, se han usado datos reales de consumo en 90 obras de edificios residenciales de distintas tipologías (13) y la metodología de Solís-Guzmán et al. (11) (12):

$$
\mathrm{HE}_{\mathrm{pa}}=\frac{\mathrm{C}}{\mathrm{Pb}} \mathrm{FE}_{\mathrm{B}}
$$

donde:

$\mathrm{HE}_{\mathrm{pa}}$ : HE ponderada del consumo de agua (hag)

$\mathrm{C}$ : consumo de agua $\left(\mathrm{m}^{3}\right)$

$\mathrm{Pb}$ : productividad de bosques ( $\left.\mathrm{m}^{3} / \mathrm{ha}\right)$. Se consideran $1.500 \mathrm{~m}^{3}$ de agua dulce por hectárea y año.

\subsubsection{Huella de superficie consumida}

La huella de ocupación directa se calculará a partir de la superficie consumida de la edificación recogida en la memoria de cada proyecto, según la expresión:

$$
\mathrm{HE}_{\mathrm{ps}}=\mathrm{S} \times \mathrm{FE}_{\mathrm{sc}}
$$

donde:

$\mathrm{HE}_{\mathrm{ps}}$ : HE ponderada de ocupación directa (hag)

S: superficie consumida (ha)

$\mathrm{FE}_{\mathrm{sc}}$ : factor de equivalencia de la superficie construida $(2,51 \mathrm{hag} / \mathrm{ha})(20)$

\section{RESULTADOS}

\subsection{Recursos empleados}

Los recursos totales empleados, por unidad de superficie y proyecto (Tabla 8), permiten obtener los resultados de HE por vivienda (parciales y total) de las diez tipologías representativas, por unidad de superficie.

\subsection{HE de las tipologías evaluadas: parciales, total y por persona}

A continuación se evalúa la HE parcial y total (Tabla 9) en función de los recursos de cada proyecto y se analizan cuáles son las fuentes de impacto que producen dicha huella y su repercusión por unidad de superficie y persona.

Los valores de superficie útil recogidas en los informes estadísticos analizados se incrementa un $20 \%$ para obtener la superficie construida.

En los resultados recogidos en la Tabla 9 se observa que la HE total es prácticamente constante a partir de 3 plantas (tipologías 4 a 10), y menor que en las viviendas unifamiliares (tipologías 1 y 2). 
Tabla 8. Recursos por unidad de superficie $\left(\mathrm{m}^{2}\right)$ de cada proyecto seleccionado.

\begin{tabular}{|c|c|c|c|c|c|c|c|c|c|c|c|}
\hline \multirow{2}{*}{\multicolumn{2}{|c|}{$\operatorname{Recursos}\left(\mathbf{u} / \mathbf{m}^{2}\right)$}} & \multicolumn{10}{|c|}{ Clasificación por tipología } \\
\hline & & \multirow{2}{*}{\begin{tabular}{|l|}
$\mathbf{1}$ \\
24,32 \\
\end{tabular}} & \multirow{2}{*}{$\begin{array}{l}2 \\
18,77 \\
\end{array}$} & \multirow{2}{*}{\begin{tabular}{|l|}
3 \\
21,40
\end{tabular}} & \multirow{2}{*}{\begin{tabular}{|l|}
4 \\
20,66
\end{tabular}} & \multirow{2}{*}{\begin{tabular}{|l|}
5 \\
20,66 \\
\end{tabular}} & \multirow{2}{*}{\begin{tabular}{|l|}
$\mathbf{6}$ \\
51,88 \\
\end{tabular}} & \multirow{2}{*}{$\begin{array}{l}7 \\
16,34\end{array}$} & \multirow{2}{*}{$\begin{array}{l}8 \\
45,27 \\
\end{array}$} & \multirow{2}{*}{\begin{tabular}{|l|}
9 \\
14,64
\end{tabular}} & \multirow{2}{*}{$\begin{array}{l}10 \\
40,31 \\
\end{array}$} \\
\hline \multirow{5}{*}{$\begin{array}{c}\text { Peso } \\
\text { materiales } \\
\text { (kg) }\end{array}$} & Acero & & & & & & & & & & \\
\hline & Hormigón & 2120,10 & 1281,23 & 1219,22 & 1262,09 & 1262,09 & 1066,17 & 1055,19 & 1007,99 & 1069,33 & 964,28 \\
\hline & Cerámico & 444,99 & 518,58 & 405,35 & 293,38 & 344,42 & 297,14 & 356,57 & 300,44 & 369,74 & 310,84 \\
\hline & Otros & 468,00 & 304,74 & 297,48 & 295,08 & 289,72 & 284,45 & 265,82 & 289,29 & 251,22 & 288,69 \\
\hline & Total & 3057,40 & 2123,32 & 1943,46 & 1871,21 & 1916,90 & 1699,65 & 1693,92 & 1642,99 & 1704,94 & 1604,12 \\
\hline \multirow{5}{*}{$\begin{array}{c}\text { Energía } \\
\text { incorporada } \\
(\mathrm{MJ})\end{array}$} & Acero & 972,67 & 750,83 & 855,81 & 826,40 & 826,40 & 2075,36 & \begin{tabular}{|r|}
653,79 \\
\end{tabular} & 1810,79 & 585,71 & 1612,41 \\
\hline & Hormigón & 2132,52 & 1288,68 & 1225,54 & 1266,60 & 1266,60 & 1070,69 & 1058,35 & 1012,50 & 1071,14 & 968,79 \\
\hline & Cerámico & 1328,71 & 1535,65 & 1198,62 & 876,79 & 1024,81 & 886,95 & 1062,92 & 896,54 & 1103,28 & 926,70 \\
\hline & Otros & 4018,06 & 3087,31 & 2463,68 & 2213,95 & 2223,22 & 2073,13 & 2255,37 & 2106,07 & 2374,03 & 2125,39 \\
\hline & Total & 8451,96 & 6662,47 & 5743,66 & 5183,75 & 5341,04 & 6106,13 & 5030,43 & 5825,90 & 5134,15 & 5633,29 \\
\hline $\begin{array}{l}\text { Mano de } \\
\text { obra }\end{array}$ & $\begin{array}{l}\text { Rendimiento } \\
\text { (h) }\end{array}$ & 13,02 & 11,62 & 9,89 & 8,99 & 9,35 & 9,06 & 9,14 & 9,05 & 0,44 & 0,41 \\
\hline \multirow[t]{2}{*}{ Maquinaria } & \begin{tabular}{|l} 
Rendimiento \\
(h)
\end{tabular} & 0,33 & 0,24 & 0,39 & 0,32 & 0,32 & 0,37 & 0,25 & 0,34 & 0,21 & 0,31 \\
\hline & Coste (€) & 2,96 & $\mathbf{1 , 9 5}$ & 6,49 & 4,95 & 4,94 & 6,84 & $\mathbf{3 , 5 2}$ & $\mathbf{5 , 8 9}$ & $\mathbf{2 , 4 1}$ & 5,17 \\
\hline \multirow{3}{*}{ Consumos } & Energía (€) & 77,19 & 54,16 & 72,21 & 43,64 & 42,67 & 42,52 & 42,89 & 43,65 & 44,19 & 43,72 \\
\hline & \begin{tabular}{|l} 
Electricidad \\
(€)
\end{tabular} & 76,59 & 53,77 & 70,91 & 42,65 & 41,68 & 41,15 & 42,19 & 42,47 & 43,71 & 42,69 \\
\hline & Agua $\left(\mathbf{m}^{3}\right)$ & 0,15 & 0,11 & 0,12 & 0,06 & 0,06 & 0,09 & 0,03 & 0,03 & 0,03 & 0,03 \\
\hline \multirow[t]{2}{*}{ Residuos (t) } & RSU & $\begin{array}{r}5,10 \\
\text { E-O3 } \\
\end{array}$ & $\begin{array}{r}4,48 \\
\text { E-O3 } \\
\end{array}$ & $\begin{array}{r}3,78 \\
\text { E-O3 } \\
\end{array}$ & $\begin{array}{r}3,48 \\
\text { E-o3 } \\
\end{array}$ & $\begin{array}{r}3,61 \\
\mathrm{E}-\mathrm{O} 3 \\
\end{array}$ & $\begin{array}{r}3,44 \\
\text { E-03 } \\
\end{array}$ & $\begin{array}{r}3,44 \\
\text { E-O3 } \\
\end{array}$ & $\begin{array}{r}3,47 \\
\text { E-03 } \\
\end{array}$ & $\begin{array}{r}3,52 \\
\mathrm{E}-03 \\
\end{array}$ & $\begin{array}{r}3,40 \\
\text { E-O3 } \\
\end{array}$ \\
\hline & RCD & 0,19 & 0,17 & 0,13 & 0,15 & 0,15 & 0,12 & 0,14 & 0,12 & 0,15 & 0,10 \\
\hline \multicolumn{2}{|c|}{ Superficie consumida (ha) } & $\begin{array}{r}1,00 \\
\text { E-04 }\end{array}$ & $\begin{array}{l}5,00 \\
\text { E-O5 }\end{array}$ & $\begin{array}{r}5,00 \\
\text { E-O5 }\end{array}$ & $\begin{array}{r}3,33 \\
\mathrm{E}-\mathrm{O} 5 \\
\end{array}$ & $\begin{array}{r}3,33 \\
\mathrm{E}-05 \\
\end{array}$ & $\begin{array}{r}2,50 \\
\text { E-05 }\end{array}$ & $\begin{array}{l}2,00 \\
\text { E-O5 }\end{array}$ & $\begin{array}{l}2,00 \\
\text { E-05 }\end{array}$ & $\begin{array}{r}1,00 \\
\text { E-05 }\end{array}$ & $\begin{array}{r}1,00 \\
\text { E-05 }\end{array}$ \\
\hline
\end{tabular}

Tabla 9. HE parciales, total y HE por persona.

\begin{tabular}{|c|c|c|c|c|c|c|c|c|c|c|}
\hline \multirow{2}{*}{ HE parcial (hag/m²) } & \multicolumn{10}{|c|}{ Clasificación por tipologías } \\
\hline & $\mathbf{1}$ & 2 & 3 & 4 & 5 & 6 & 7 & 8 & 9 & 10 \\
\hline Energía & 0,26 & 0,20 & 0,20 & 0,16 & 0,16 & 0,17 & 0,16 & 0,17 & 0,16 & 0,17 \\
\hline Bosques & $\begin{array}{r}1,28 \\
\text { E-04 }\end{array}$ & $\begin{array}{l}8,96 \\
\text { E-05 }\end{array}$ & $\begin{array}{r}1,03 \\
\text { E-04 }\end{array}$ & $\begin{array}{r}4,97 \\
\text { E-05 }\end{array}$ & $\begin{array}{r}4,97 \\
\text { E-05 }\end{array}$ & $\begin{array}{r}7,33 \\
\text { E-05 }\end{array}$ & $\begin{array}{l}2,52 \\
\text { E-05 }\end{array}$ & $\begin{array}{l}2,85 \\
\text { E-O5 }\end{array}$ & $\begin{array}{l}2,43 \\
\text { E-O5 }\end{array}$ & $\begin{array}{r}2,54 \\
\text { E-O5 }\end{array}$ \\
\hline Pastos & 0,05 & 0,04 & 0,04 & 0,03 & 0,04 & 0,03 & 0,04 & 0,03 & 0,04 & 0,03 \\
\hline Mar & 0,03 & 0,03 & 0,03 & 0,02 & 0,02 & 0,02 & 0,02 & 0,02 & 0,02 & 0,02 \\
\hline Cultivos & 0,02 & 0,02 & 0,01 & 0,01 & 0,01 & 0,01 & 0,01 & 0,01 & 0,01 & 0,01 \\
\hline Superficie consumida & $\begin{array}{r}2,51 \\
\text { E-04 }\end{array}$ & $\begin{array}{r}1,26 \\
\text { E-04 }\end{array}$ & $\begin{array}{r}1,26 \\
\text { E-04 }\end{array}$ & $\begin{array}{r}8,37 \\
\text { E-05 }\end{array}$ & $\begin{array}{r}8,37 \\
\text { E-05 }\end{array}$ & $\begin{array}{l}6,28 \\
\text { E-05 }\end{array}$ & $\begin{array}{l}5,02 \\
\text { E-05 }\end{array}$ & $\begin{array}{l}5,02 \\
\text { E-05 }\end{array}$ & $\begin{array}{r}2,51 \\
\text { E-05 }\end{array}$ & $\begin{array}{r}2,51 \\
\text { E-05 }\end{array}$ \\
\hline HE Total (hag/m²) & 0,362 & 0,284 & 0,277 & 0,232 & 0,237 & 0,243 & 0,230 & 0,241 & 0,236 & 0,237 \\
\hline $\begin{array}{l}\text { Superficie constr. por } \\
\text { viviendas }\left(\mathrm{m}^{2}\right)\end{array}$ & 217,20 & 171,60 & 86,40 & 86,40 & 86,40 & 84,00 & 86,40 & 86,4 & 86,40 & 86,40 \\
\hline $\begin{array}{c}\text { Número } \\
\text { dormitorios }\end{array}$ & 5 & 5 & 3 & 3 & 3 & 3 & 3 & 3 & 3 & 3 \\
\hline Número personas s/CTE & 6 & 6 & 4 & 4 & 4 & 4 & 4 & 4 & 4 & 4 \\
\hline Número personas por $\mathbf{m}^{2}$ & 0,03 & 0,03 & 0,05 & 0,05 & 0,05 & 0,05 & 0,05 & 0,05 & 0,05 & 0,05 \\
\hline HE por persona (hag) & 13,11 & 8,13 & 5,97 & 5,01 & 5,12 & 5,10 & 4,97 & 5,20 & 5,09 & 5,13 \\
\hline
\end{tabular}


La HE producida por la maquinaria de motor de combustión es significativamente baja en la construcción, ya que su uso se limita a las primeras fases de la obra (movimiento de tierras y cimentación), mientras que en el resto de la obra la maquinaria empleada consume principalmente energía eléctrica.

La huella de la energía es la más importante en todas las tipologías, la cual engloba a su vez el impacto producido por la energía incorporada de los materiales de construcción, la energía en el procesado de los alimentos, movilidad de los trabajadores, electricidad y residuos.

Por otro lado, agrupando la HE por fuentes de impacto, la mayor huella la producen los materiales (45\%) y los alimentos (34\%) consumidos por la mano de obra (energía, cultivos, mar y pastos), que controlan alrededor del $80 \%$ de la HE total. La electricidad consumida en la obra también es relevante (18\%). La HE de los residuos dividida entre RCD (70 \% aprox.), y RSU (30 \%) también es apreciable (3\%).

Maquinaria, movilidad y superficie consumida tienen valores mínimos dentro de la HE total, al igual que la huella del agua (Tabla 9).

La HE provocada por la mano de obra se divide en los RSU, la movilidad (menos del $1 \%$ ) y la alimentación (pastos, mar productivo, cultivos y energía), siendo ésta última la que representa casi toda la HE analizada (Tabla 10).
Como parte del análisis se representa la HE de las personas que habitan cada vivienda (Tabla 9). Los cálculos se basan en el documento DB-HS3 del CTE (29) según el número de dormitorios por vivienda (16), definidos para cada tipología. Los resultados siguen la misma tendencia que la $\mathrm{HE}$ por vivienda, es decir, las personas que habitan en viviendas de tipologías 1 y 2 que corresponden a viviendas unifamiliares tienen mayor $\mathrm{HE}$ que el resto, que habitan viviendas plurifamiliares.

\subsection{HE por capítulos del proyecto}

Para poder realizar el análisis de las características constructivas y los sistemas empleados, se amplía el modelo desglosando los recursos según los capítulos de la clasificación sistemática del BCCA (15). Seleccionadas tres tipologías $-3,7$ y 9- definidas en las Tablas 3 y 4, se realiza la comparativa (Figura 3), según el número de plantas, que en este caso corresponden a edificios de viviendas de 2, 5 y 10 plantas sobre rasante y una planta bajo rasante, respectivamente. Los capítulos que determinan la $\mathrm{HE}$ de cada proyecto son estructuras, albañilería, revestimientos y cimentaciones, los restantes tienen mucho menor impacto. En la fase de estructuras el mayor impacto lo producen los materiales, y en los revestimientos la mano de obra. Los capítulos de cimentaciones y estructura tienen una mayor huella en viviendas bajas, y las instalaciones y revestimientos en viviendas con mayor número de plantas. Se identifican así, en fase de proyecto los procesos de mayor impacto.

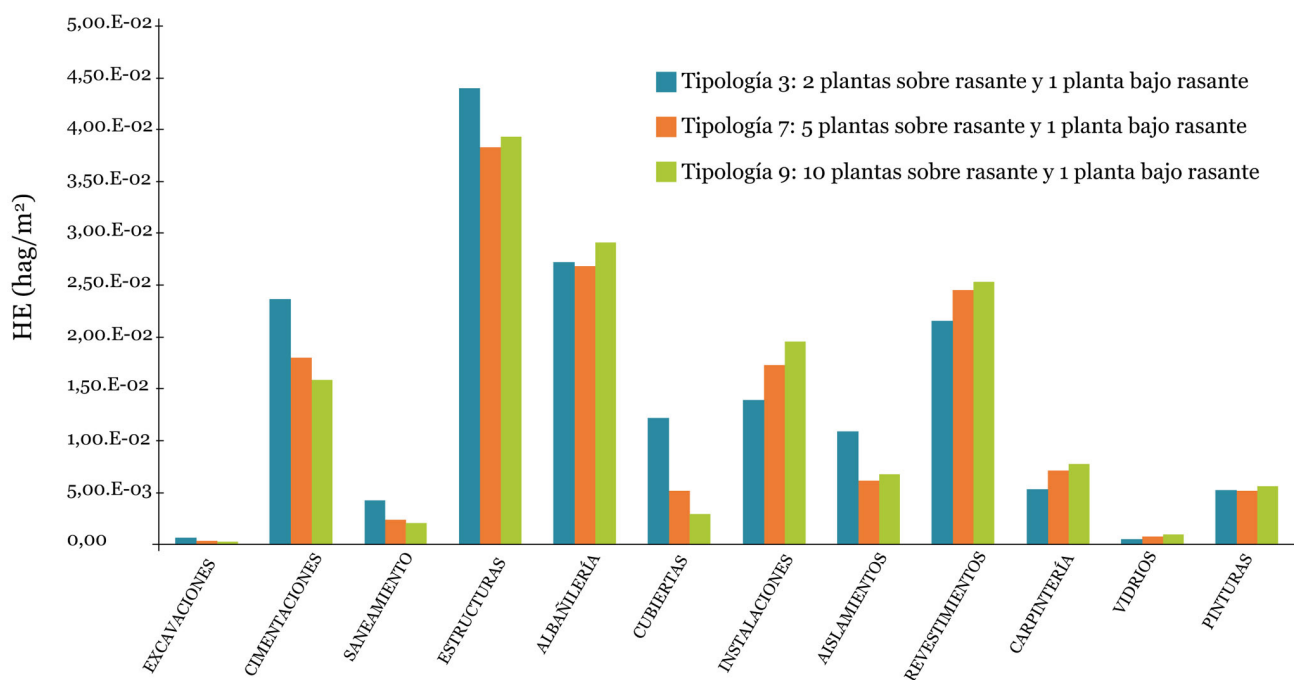

Figura 3. HE por capítulos según fases de proyecto.

Tabla 10. HE de la mano de obra.

\begin{tabular}{|c|c|c|c|c|c|c|c|c|c|c|}
\hline \multirow{3}{*}{$\begin{array}{c}\text { Impacto } \\
\text { Mano de obra } \\
\left(\mathbf{h a g} / \mathbf{m}^{2}\right)\end{array}$} & \multicolumn{10}{|c|}{ Huella Ecológica de la mano de obra en la edificación (hag/m²) } \\
\hline & \multicolumn{10}{|c|}{ Clasificación por tipología } \\
\hline & $\mathbf{1}$ & 2 & 3 & 4 & 5 & 6 & 7 & 8 & 9 & 10 \\
\hline Alimentos & 0,115 & 0,098 & 0,065 & 0,08 & 0,083 & 0,081 & 0,083 & 0,082 & 0,086 & 0,082 \\
\hline Movilidad & $\begin{array}{r}3,87 \\
\text { E-06 }\end{array}$ & $\begin{array}{r}3,27 \\
\text { E-06 } \\
\end{array}$ & $\begin{array}{r}2,18 \\
\text { E-06 }\end{array}$ & $\begin{array}{r}2,67 \\
\text { E-06 } \\
\end{array}$ & $\begin{array}{r}2,83 \\
\text { E-06 }\end{array}$ & $\begin{array}{r}2,72 \\
\text { E-06 } \\
\end{array}$ & $\begin{array}{r}2,77 \\
\text { E-06 } \\
\end{array}$ & $\begin{array}{r}2,73 \\
\text { E-06 } \\
\end{array}$ & $\begin{array}{l}2,88 \\
\text { E-06 }\end{array}$ & $\begin{array}{r}2,75 \\
\text { E-06 }\end{array}$ \\
\hline Residuos RSU & 0,003 & 0,002 & 0,002 & 0,002 & 0,002 & 0,002 & 0,002 & 0,002 & 0,002 & 0,002 \\
\hline $\begin{array}{l}\text { HE total } \\
\text { mano de obra }\left(\mathrm{hag} / \mathrm{m}^{2}\right)\end{array}$ & $\mathbf{0 , 1 1 8}$ & $\mathbf{0 , 1 0 1}$ & $\mathbf{0 , 0 6 7}$ & $\mathbf{0 , 0 8 2}$ & $\mathbf{0 , 0 8 5}$ & $\mathbf{0 , 0 8 3}$ & $\mathbf{0 , 0 8 5}$ & $\mathbf{0 , 0 8 4}$ & $\mathbf{0 , 0 8 8}$ & $\mathbf{0 , 0 8 4}$ \\
\hline Porcentaje sobre HE total & $33 \%$ & $35 \%$ & $32 \%$ & $36 \%$ & $36 \%$ & $34 \%$ & $37 \%$ & $35 \%$ & $38 \%$ & $36 \%$ \\
\hline
\end{tabular}


Analizando en profundidad estos cuatro capítulos, para el caso de la cimentación los materiales suponen el $85 \%$ sobre el total de los recursos (materiales, mano de obra y maquinaria), siendo el tipo 3 (2 plantas) el que tiene mayor HE, y el impacto mayor corresponde al hormigón y al acero de las armaduras.

En el caso del capítulo de estructuras los materiales suponen un $78 \%$ del total de recursos, el proyecto de mayor huella es el tipo 3, y los de mayor impacto son el acero de las armaduras, el cemento de las bovedillas y el hormigón armado, en ese orden.

Para el capítulo de albañilería, los materiales y la mano de obra tienen prácticamente el mismo impacto dentro del total, 57 y $47 \%$, respectivamente. En esta fase el proyecto de mayor HE es el tipo 9 (10 plantas), y el impacto de los materiales es debido a la cerámica de los ladrillos.

Por último, en el caso del capítulo de revestimientos, la mano de obra es el recurso que genera mayor impacto (85\%), frente a los materiales (15\%). El proyecto tipo 9 es el de mayor huella, y la mano de obra empleada es casi el doble que en la fase de albañilería. Los materiales con mayor impacto son el hormigón en masa de las soleras, la cerámica de los azulejos y solería, y por último el mortero de perlita del guarnecido y enlucido.

\subsection{HE en España: total y por persona}

Al aplicar los datos obtenidos de HE de cada una de las tipologías a las Estadísticas de Fomento sobre el número de viviendas construidas (Tabla 1) y con los porcentajes de cada una de ellas (Tabla 2) obtenemos la HE total construida por año y la HE por persona en el período 2007-2010 (Tabla 11). Las columnas de porcentajes corresponden a las viviendas construidas de cada tipología para cada año.

La HE de la edificación de tipo residencial en España por persona es muy similar cada año, sin embargo en el año 2010 (Tabla 11) es mayor que en los anteriores, debido a que existe un mayor porcentaje de viviendas construidas de tipo unifamiliar.
Para establecer una comparativa con la HE a nivel país, se realizan las siguientes consideraciones:

- Se calcula el valor medio de los valores de HE total por persona (5,84 hag/persona).

- Se define una vida útil estimada para los edificios estudiados (50 años), lo que nos da una HE de 0,1168 hag/persona/año.

- Comparamos este resultado con los datos de HE y biocapacidad disponibles para España (30) (4,74 y 1,46 hag/persona/año respectivamente), obteniendo que un habitante consume anualmente el 2,46 \% de su HE y el $8 \%$ de su biocapacidad disponible en la construcción de esas viviendas.

\section{CONCLUSIONES}

El marco de referencia de la HE ha sido definido gracias a la evaluación en informes estadísticos de las tipologías y los sistemas constructivos empleados en España entre 2007 y 2010. $\mathrm{La} \mathrm{HE}$ en la fase de construcción de edificios residenciales ha sido evaluada satisfactoriamente, concluyendo que el análisis de HE es sensible a cambios en las características de los edificios, como el número de plantas o la tipología, y que estos cambios afectan significativamente a los resultados.

La HE por metro cuadrado construido en viviendas de tipo unifamiliar es muy superior a la de las viviendas pertenecientes a edificios plurifamiliares de tres plantas o más, y ésta permanece prácticamente constante a partir de tres plantas. La mano de obra y los materiales tienen un significativo impacto en la HE en todos los casos analizados.

También ha sido posible la evaluación de la HE por fases de proyecto, arrojando que la estructura o la albañilería tienen una gran repercusión en el impacto total. Analizando en detalle las distintas fases, en la cimentación los materiales suponen un $85 \%$ sobre el total de los recursos, con el hormigón y el acero de las armaduras como elementos principales; y el proyecto de mayor impacto por metro cuadrado es el de 2 plantas. En la fase de estructuras, los materiales tienen un impacto similar, y el proyecto de mayor huella es el de 2 plantas, y son el acero de las armaduras, el cemento de las bove-

Tabla 11. HE total y por persona de las viviendas construidas en España en el período 2007-2010.

\begin{tabular}{|c|c|c|c|c|c|c|c|c|}
\hline \multirow{2}{*}{ Tipologías } & \multicolumn{8}{|c|}{ HE total de viviendas construidas por año (hag) } \\
\hline & 2007 & $\%$ & 2008 & $\%$ & 2009 & $\%$ & 2010 & $\%$ \\
\hline $\mathbf{1}$ & $1.854 \cdot 339$ & 3,7 & 1.104 .642 & 5,23 & 610.377 & 5,94 & 588.350 & 8,16 \\
\hline 2 & 3.703 .145 & 12,0 & 1.558 .104 & 11,90 & 674.505 & 10,60 & 587.547 & 13,15 \\
\hline 3 & 1.361 .253 & 12,0 & 572.749 & 11,90 & 247.944 & 10,60 & 215.978 & 13,15 \\
\hline 4 & $1.449 \cdot 371$ & 11,4 & 527.719 & 9,82 & 250.510 & 9,58 & 136.512 & 7,44 \\
\hline 5 & 1.481 .228 & 11,4 & 539.319 & 9,82 & 256.016 & 9,58 & 139.512 & 7,44 \\
\hline 6 & 1.429 .225 & 11,0 & 590.182 & 10,77 & 262.046 & 9,83 & 174.590 & 9,33 \\
\hline 7 & 1.391 .032 & 11,0 & 574.411 & 10,77 & 255.043 & 9,83 & 169.925 & 9,33 \\
\hline 8 & $1.455 \cdot 391$ & 11,0 & 600.987 & 10,77 & 266.843 & 9,83 & 177.787 & 9,33 \\
\hline 9 & 1.057 .498 & 8,2 & 519.827 & 9,51 & 321.722 & 12,10 & 211.559 & 11,33 \\
\hline 10 & 1.064 .433 & 8,2 & 523.237 & 9,51 & 323.832 & 12,10 & 212.946 & 11,33 \\
\hline HE total por año (hag) & 16.246 .913 & 100 & 7.111 .178 & 100 & 3.468 .838 & 100 & 2.614 .706 & 100 \\
\hline $\begin{array}{c}\text { HE total por persona } \\
\text { y año (hag) }\end{array}$ & \multicolumn{2}{|l|}{5,68} & \multicolumn{2}{|c|}{5,80} & \multicolumn{2}{|l|}{5,82} & \multicolumn{2}{|c|}{6,06} \\
\hline
\end{tabular}


dillas y el hormigón los de mayor impacto. En ambas fases, por tanto, se deben seleccionar materiales alternativos (producidos con energías limpias, reutilizados o reciclados) con menor energía incorporada para reducir la huella.

Existen fases como la de albañilería donde tanto los materiales como la mano de obra tienen prácticamente el mismo impacto dentro del total, el proyecto de mayor HE es el de 10 plantas, debido a los ladrillos y la intensidad de mano de obra.

Por otro lado, en la fase de revestimientos la mano de obra es el recurso que genera mayor impacto (85 \%). El proyecto de mayor huella por metro cuadrado es el de 10 plantas. En cuanto a los materiales que tienen mayor impacto están el hormigón en masa de las soleras, la cerámica de azulejos y solería y el mortero de perlita del guarnecido y enlucido.

También se ha evaluado la HE total de los edificios residenciales construidos en España en 2007-2010, constatando la reducción paulatina de dicha huella, debido a la menor actividad constructora. Sin embargo, la HE por persona ha aumentado en dicho período, debido al incremento del porcentaje de viviendas unifamiliares construidas.

En definitiva, se ha demostrado la validez del indicador HE para su aplicación a cualquier escala, desde la planetaria hasta la de los edificios, e incluso siendo sensible a cambios en los sistemas constructivos.
En desarrollos futuros se evaluarán otras etapas del ciclo de vida de los edificios, como son el uso y mantenimiento, las renovaciones y la demolición, que deben tener una repercusión importante en la toma de decisiones para una construcción más sostenible. Otro aspecto a analizar, es que las tipologías más construidas en España, y por tanto los proyectos estudiados, no tienen instalaciones de energías renovables o de tratamiento de aguas residuales, lo que es fundamental para desarrollar una menor huella en el ciclo de vida de los edificios, y que debe incorporarse al desarrollo del modelo de HE propuesto.

Para finalizar, independientemente de las conclusiones expuestas, entendemos que la investigación realizada transmite la idea de cómo puede influir con mayor o menor intensidad los diferentes aspectos caracterizadores de las viviendas en la cuantificación de la HE. Por tanto, sirve de reflexión como un obligado modo de situarse, tanto ante la proyección de nueva planta como ante las actuaciones rehabilitadoras (actividad ésta última que tiende a desarrollarse en los próximos tiempos), para ir caminando en producir la menor HE posible en este sector tan influyente.

\section{AGRADECIMIENTOS}

Ministerio de Innovación y Ciencia, por la concesión del proyecto I+D+i: Evaluación de la huella ecológica de la edificación del sector residencial en España (EVALHED) (BIA201125812).

\section{REFERENCIAS}

(1) Sabaté, J., Peters, C. (2011). Una visión holística de la reducción del impacto ambiental en edificios del área del Mediterráneo. Informes de la Construcción, 63(EXTRA): 73-87, doi: http://dx.doi.org/10.3989/ic.11.066.

(2) Wadel, G., Avellaneda, J., Cuchí, A. (2010). La sostenibilidad en la arquitectura industrializada: cerrando el ciclo de los materiales. Informes de la Construcción, 62(517): 37-51, doi: http://dx.doi.org/10.3989/ic.09.067.

(3) Ministerio de Fomento. (2012). Total de viviendas por comunidades autónomas y provincias 2002-2011. Madrid, España. http://www.fomento.gob.es/BE2/?nivel=2\&orden=33000000.

(4) Zabalza-Bribián, I., Valero-Capilla, A., Aranda-Usón, A. (2011). Life cycle assessment of building materials: Comparative analysis of energy and environmental impacts and evaluation of the eco-efficiency improvement potential. Building and Environment, 46(5): 1133-1140, doi: http://dx.doi.org/10.1016/j.buildenv.2010.12.002.

(5) Meillaud, F., Gay, J., Brown, M.T. (2005). Evaluation of a building using the emergy method. Solar Energy, 79(2): 204212, doi: http://dx.doi.org/10.1016/j.solener.2004.11.003.

(6) Sinivuori, P., Saari, A. (2006). MIPS analysis of natural resource consumption in two university buildings. Building and Environment, 41(5): 657-668, doi: http://dx.doi.org/10.1016/j.buildenv.2005.02.022.

(7) Chambers, N., Simmons, C., Wackernagel, M. (2004). Sharing Nature's Interest: Ecological Footprint as an Indicator of Sustainability. London, Great Britain: Sterling Earthscan.

(8) Wackernagel, M., Rees, W. (1996). Our Ecological Footprint: Reducing Human Impact on the Earth. Gabriola Island, British Columbia: New Society.

(9) Solís-Guzman, J., Martínez-Rocamora, A., Marrero, M. (2014). Methodology for determining the carbon footprint of the construction of residential buildings. En Muthu, S.S. (Ed), Assessment of Carbon Footprint in Different Industrial Sectors, (Vol. 1, pp. 49-83). Singapore: Springer Science. Doi: http://dx.doi.org/10.1007/978-981-4560-41-2_3.

(10) Galli, A., Wiedmann, T., Ercin, E., Knoblauch, D., Ewing, B., Giljum, S. (2012). Integrating Ecological, Carbon and Water footprint into a "Footprint Family" of indicators: Definition and role in tracking human pressure on the planet. Ecological Indicators, 16: 100-112, doi: http://dx.doi.org/10.1016/j.ecolind.2011.06.017.

(11) Solís-Guzmán, J., Marrero, M., Ramírez de Arellano, A. (2013). Methodology for determining the ecological footprint of the construction of residential buildings in Andalusia (Spain). Ecological Indicators, 25: 239-249, doi: http://dx.doi. org/10.1016/j.ecolind.2012.10.008.

(12) Solís-Guzmán, J. (2011). Evaluación de la huella ecológica del sector edificación (uso residencial) en la comunidad andaluza (Tesis doctoral). Universidad de Sevilla, España. http://fondosdigitales.us.es/tesis/tesis/2051/evaluacion-de-lahuella-ecologica-del-sector-edificacion-uso-residencial-en-la-comunidad-andaluza/.

(13) González-Vallejo, P., Marrero, M., Solís-Guzmán, J. (2015). The ecological footprint of dwelling construction in Spain. Ecological Indicators, 52: 75-84, doi: http://dx.doi.org/10.1016/j.ecolind.2014.11.016. 
(14) Marrero, M., Ramirez-de Arellano, A. (2010). The building cost system in Andalusia: application to construction and demolition waste management. Construction Management and Economics, 28(5): 495-507, doi: http://dx.doi. org/10.1080/01446191003735500.

(15) ConsejeríadeObrasPúblicasyViviendadelaJuntadeAndalucía.BasedeCostesdelaConstruccióndeAndalucía.(2010).http:// www.juntadeandalucia.es/fomentoyvivienda/portal-web/web/areas/vivienda/texto/28c8aa4d-2483-11eo-a6d19169d73od750.

(16) MinisteriodeFomento.(2011).Construccióndeedificios20o6-2010.Datosrecogidosdelaslicenciasdeobras.Madrid,España. http://www.fomento.gob.es/NR/rdonlyres/32B384B3-DFF4-40B6-ADA2-870AADC7E077/106601/Construccion Edificios2010.pdf.

(17) Domenech-Quesada, J.L. (2007). Huella Ecológica y Desarrollo Sostenible. Madrid, España: AENOR.

(18) Instituto para la Diversificación y Ahorro de la Energía (IDAE). (2011). Guía Práctica de la Energía. Madrid, España. http://www.idae.es/index.php/mod.pags/mem.detalle/relcategoria.1161/id.542/relmenu.64.

(19) Acosta-Bono, G., González-Daimiel, J., Calvo-Salazar, M., Sancho-Royo, F. (2001). Estimación de la Huella Ecológica en Andalucía y Aplicación a la Aglomeración Urbana de Sevilla. Sevilla, España: DGTOTyU, Consejería de Obras Públicas de la Junta de Andalucía. http://infodigital.opandalucia.es/bvial/handle/10326/974.

(20) Ewing, B., Moore, D., Goldfinger, S., Oursler, A., Reed, A., Wackernagel, M. (2010). The Ecological Footprint Atlas 2010. Oakland: Global Footprint Network. http://www.footprintnetwork.org/images/uploads/Ecological_Footprint_ Atlas_2010.pdf.

(21) Consejería de Medio Ambiente de Andalucía. (2009). Informe de Medio Ambiente 20o8. http://www.juntadeandalucia. es/medioambiente/site/ima.

(22) Ministerio de Medio Ambiente. (2001). Plan Nacional de Residuos de Construcción y Demolición 2001-2006. Madrid, España.

(23) Marrero, M., Martínez-Escobar, L., Mercader-Moyano, P., Leiva, C. (2013). Minimización del impacto ambiental en la ejecución de fachadas mediante el empleo de materiales reciclados. Informes de la Construcción, 65(529): 89-97, doi: http://dx.doi.org/10.3989/ic.11.034.

(24) Corporación de Reservas Estratégicas de Productos Petrolíferos (CORES). (2013). Informe estadístico anual. Año 2012. http://www.cores.es/es/node/101.

(25) Ministerio de Industria. (2012). Estructura de generación eléctrica en España. La Energía en España 2012. http:// www.minetur.gob.es/energia/balances/Balances/LibrosEnergia/Energia_en_Espana-2012.pdf.

(26) Wackernagel, M., Dholakia, R., Deumling, D., Richardson, D. (2000). Redefining Progress, Assess your Household's Ecological Footprintv 2.o. http://cads.mty.itesm.mx/lds/huella.xls.

(27) Ramirez de Arellano-Agudo, A., Solís-Guzmán, J., Pérez-Monge, J. (2008). Software de Evaluación de RCD para Tramitación de Licencias Municipales. Sevilla, España: Universidad de Sevilla.

(28) Marañón, E., Iregui, G., Domenech, J.L., Fernández-Nava, Y., González, M. (2008). Propuesta de índices de conversión para la obtención de la huella de los residuos y los vertidos. Observatorio Iberoamericano del Desarrollo Local y la Economía Social, 2(4).

(29) Ministerio de Vivienda. (2006). Código Técnico de la Edificación. Madrid, España.

(30) WWF. (2012). Informe Planeta Vivo 2012. Gland, Suiza: WWF Internacional. 